\title{
Electro-optic modulation in slotted resonant photonic crystal heterostructures
}

\author{
Jan Hendrik Wülbern, ${ }^{1, a)}$ Jan Hampe, ${ }^{1}$ Alexander Petrov, ${ }^{1}$ Manfred Eich, ${ }^{1}$ Jingdong Luo, ${ }^{2}$ \\ Alex K.-Y. Jen, ${ }^{2}$ Andrea Di Falco, ${ }^{3}$ Thomas F. Krauss, ${ }^{3}$ and Jürgen Bruns ${ }^{4}$ \\ ${ }^{1}$ Hamburg University of Technology, Eissendorfer Str. 38, D-21073 Hamburg, Germany \\ ${ }^{2}$ Department of Materials Science and Engineering, University of Washington, Seattle, \\ Washington 98194-2120, USA \\ ${ }^{3}$ School of Physics and Astronomy, University of St Andrews, North Haugh, St Andrews KY16 9AJ, Scotland \\ ${ }^{4}$ Fachgebiet Hochfrequenztechnik, Technische Universität Berlin, D-10587 Berlin, Germany
}

(Received 14 May 2009; accepted 28 May 2009; published online 17 June 2009)

Two dimensional photonic crystal waveguides in high index materials enable integrated optical devices with an extremely small geometrical footprint on the scale of micrometers. Slotted waveguides are based on the guiding of light in low refractive index materials and a field enhancement in this particular region of the device. In this letter we experimentally demonstrate electro-optic modulation in slotted photonic crystal waveguides based on silicon-on-insulator substrates covered and infiltrated with nonlinear optical polymers. A photonic crystal heterostructure is used to create a cavity, while simultaneously serving as an electrical connection from the slot to the metal electrodes that carry the modulation signal. (c) 2009 American Institute of Physics. [DOI: $10.1063 / 1.3156033$ ]

Electrically driven optical modulation in silicon photonics typically relies on interactions between the optical mode and a free carrier plasma via either carrier depletion, injection, or accumulation. ${ }^{1-3}$ The achievable modulation speed using these methods is limited by the time constants related to the injection or removal of carriers from the optical waveguide. In contrast, modulation via nonlinear optical (NLO) polymers accesses the electronic polarization of the organic molecules, which allows extremely high modulation speeds extending up to frequencies in the terahertz range. ${ }^{4}$ Furthermore, molecular engineering of organic molecules has led to extremely high Pockels-coefficients in polymers exceeding $300 \mathrm{pm} / \mathrm{V}$, ${ }^{5}$ which is ten times the value available in lithiumniobate, the standard inorganic material used in electro-optic (EO) applications. Photonic devices based on a hybrid material system merging silicon and polymer are therefore attractive since they combine the strong light confining abilities of silicon with the superior NLO properties of polymers.

All-optical and EO-modulation in such hybrid silicon and NLO-polymer systems has been demonstrated for slotted photonic wire based Mach-Zehnder and ring-resonator modulators. ${ }^{6-8}$ Concepts based on slotted photonic crystal $(\mathrm{PhC})$ waveguides can exploit slow light mechanisms or high quality factor cavities to achieve very compact device dimensions and have been discussed recently. ${ }^{9-11}$ We propose a concept using a double heterostructure cavity ${ }^{11-13}$ in a slotted silicon PhC waveguide, infiltrated with NLO-polymer to operate as an EO-modulator. Figure 1 shows a scanning electron micrograph of the structure. The $150 \mathrm{~nm}$ wide slot in the center of the waveguide is filled with NLO-polymer ( $n_{\text {poly }}$ $=1.63)$ and the strong overlap between the optical field and the polymer makes the effective index of the propagating mode very sensitive to any refractive index changes in EOpolymer. The $\mathrm{PhC}$ features a background doping density of $10^{15} \mathrm{~cm}^{-1}$ and therefore serves as an electrical conductor

${ }^{a)}$ Electronic mail: jan.wuelbern@tu-harburg.de. from the metallic contact pads to the slot, while keeping the optical field away from the metal regions and hence preventing additional losses. The double heterostructure design of the $\mathrm{PhC}(a=410 \mathrm{~nm}, r / a=0.3$ and elongated cavity lattice constant $a_{\text {cav }}=420 \mathrm{~nm}$, see Fig. 1) allows the formation of a cavity with quality factor of 50000 or higher ${ }^{12}$ in not infiltrated structures and $Q$ factors of 10000 in NLO-polymer filled structures ${ }^{11}$ while maintaining very compact geometric dimensions on the micrometer scale. To facilitate coupling from the $\mathrm{PhC}$ waveguide to the slotted wire waveguide an injector section with an increased lattice constant $a_{\text {inj }}$ $=450 \mathrm{~nm}$ is introduced. ${ }^{11}$ Furthermore, a tapered coupler transition serves to couple the light between the unslotted ridge waveguide and the slotted waveguide. ${ }^{14}$

The devices were fabricated using an etching mask of ZEP-520 electron beam resist patterned by electron beam

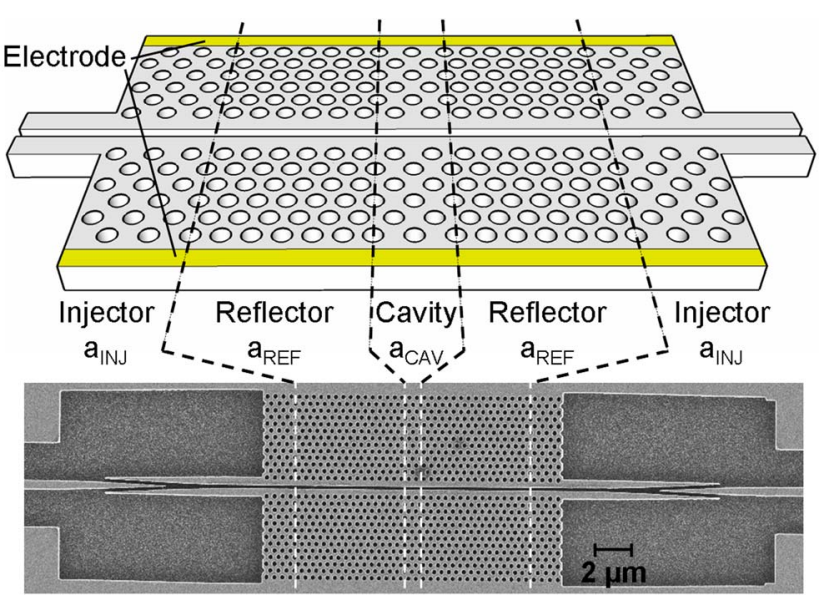

FIG. 1. (Color online) Top: schematic of the slotted PhC heterostructure modulator. The relevant electric field is in the plane of the slab across the slotted region. It is induced by the modulation voltage, which is applied to the metal electrodes alongside the $\mathrm{PhC}$ waveguide. Bottom: a scanning electron micrograph of the investigated slotted $\mathrm{PhC}$ resonators. 


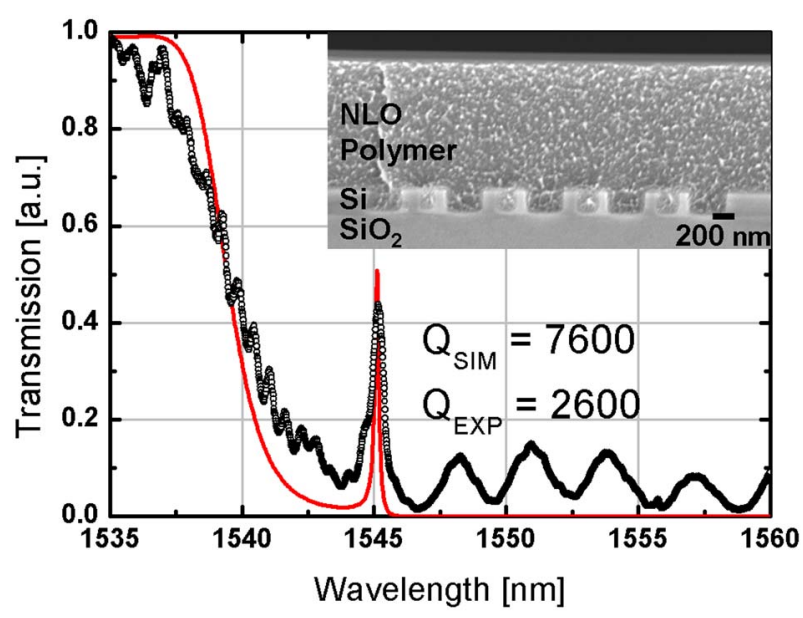

FIG. 2. (Color online) Calculated (red line) and experimental (open symbols) transmission spectra of the hybrid SOI and polymer double heterostructure resonator. The inset shows a scanning electron micrograph of the PhC structure completely infiltrated with NLO-polymer.

lithography and subsequent inductively coupled plasma etching on a Soitec silicon-on-insulator (SOI) wafer with a $220 \mathrm{~nm}$ thick Si layer on $2 \mu \mathrm{m}$ buried oxide. The aluminum contact pads serving as electrodes were deposited using a shadow mask and electron beam evaporation. Finally, the samples were covered by a polymer with strong second order NLO response consisting of a guest host system of EO-active chromophore AJ-CKL1 doped 25 wt \% into amorphous polycarbonate (poly[bisphenol- $A$-carbonate $]_{x}$-co[4, 4'-(3,3,5-trimethylcyclohexylidene)diphenol carbonate]) (APC). ${ }^{15}$ The polymer was deposited from solution (6 wt \% in cyclopentanone) and subsequently baked at $80{ }^{\circ} \mathrm{C}$ under vacuum for $12 \mathrm{~h}$ to remove residual solvent. The complete infiltration of the nanostructures with NLO-polymer is shown in the inset of Fig. 2.

To align the NLO-chromophores in the slotted region noncentrosymmetrically, the sample was heated to the polymer glass temperature $\left(T_{g}=145^{\circ} \mathrm{C}\right)$ while applying $20 \mathrm{~V}$ poling voltage to the electrodes, resulting in a poling field in the slot of approximately $130 \mathrm{~V} / \mu \mathrm{m}$. After reaching the glass temperature the sample was rapidly cooled down to room temperature. At room temperature the poling voltage was switched off, thus freezing the molecular orientation of the NLO-chromophores in the polymer matrix and preserving the macroscopic EO-response in the absence of the poling field.

The devices were characterized in an end-fire setup using a broadband source (1525-1605 nm bandwidth) and an optical spectrum analyzer. For the EO-modulation measurements, a tunable laser source was employed and the signal detected with a photodiode. The photodiode was connected to a lock-in amplifier which also supplied the electric modulation signal to the device. This technique enables the separate observation of the Pockels effect and electrical Kerr effect, which have linear and quadratic dependency on the electric field, respectively. For simplicity modulation experiments were carried out at a frequency of $f_{\bmod }=5 \mathrm{kHz}$.

We examined the transmission characteristics of a slotted heterostructure. Figure 2 shows a clear resonance peak with a quality factor of 2600. We compared this spectrum to numerical results obtained from the finite integration technique code of CST Microwave Studio, ${ }^{16}$ which are superimposed

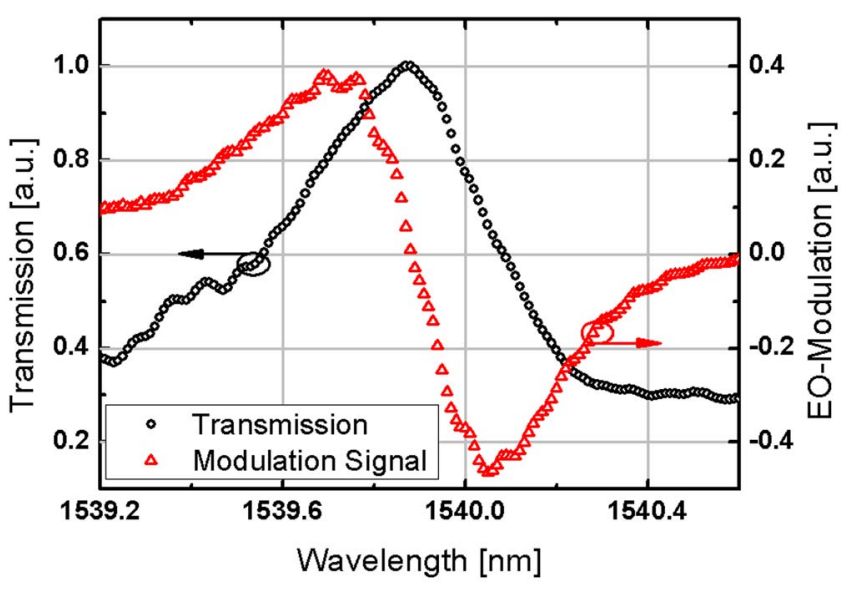

FIG. 3. (Color online) Spectral transmission (black circles) and modulation response (red triangles) of a slotted $\mathrm{PhC}$ heterostructure after infiltration with APC/AJ-CKL1 guest host polymer and after high electric field poling. The magnitude of the detected modulated signal corresponds qualitatively to the first derivative of the transmission spectrum.

on the experimental curve in Fig. 2. In order to obtain the good fit displayed in the figure, the calculated spectrum was redshifted by $1.6 \%$ in wavelength compared to the experimental results, which is a reasonable deviation given experimental uncertainties. ${ }^{17}$ The numerically obtained $Q$-factor is approximately three times higher than the one obtained in our experiments, which is most likely due to fabrication imperfections.

A spectral scan of the optical transmission and EOmodulation behavior of a second $\mathrm{PhC}$ heterostructure is shown in Fig. 3. The linewidth of the resonance peak in this sample was approximately $\Delta \lambda=0.7 \mathrm{~nm}$, which corresponds to a quality factor of $Q=2200$. The magnitude of the modulated signal as a function of wavelength is expected to be directly proportional to the first derivative of the transmission spectrum of the modulator for small perturbations of the effective refractive index. This is confirmed by the spectral response of the detected modulation signal in Fig. 3. Most noteworthy is the zero crossing of the modulated signal at the resonance frequency and the accompanying phase shift in $\pi$ as well as the two prominent extremum points on either side of the resonance frequency.

As mentioned before the detection by virtue of the lock-in technique allows the separate detection of the contributions of the Pockels and electrical Kerr effect. For this experiment the output of the laser source was tuned to the wavelength of the maximum modulation response found in Fig. 3 at $1540 \mathrm{~nm}$. The modulated signal at the first and second harmonic of the modulation frequency were then recorded as a function of the applied voltage to the electrodes, which is directly proportional to the quasistatic field in the polymer filled slot. The results are presented in Fig. 4. As predicted the modulated signal shows a linear and quadratic dependence on the quasistatic electric field amplitude at the first and second harmonic of the modulation frequency, respectively. This confirms the observation of EO-modulation due to the Pockels and electrical Kerr effect. Finally, the sample was thermally annealed to randomize the orientation of the NLO-chromophores in the polymer host matrix and the above experiments were repeated. After the thermal treatment the EO-susceptibility had been erased and thus the sample did not show any further EO-response, which con- 


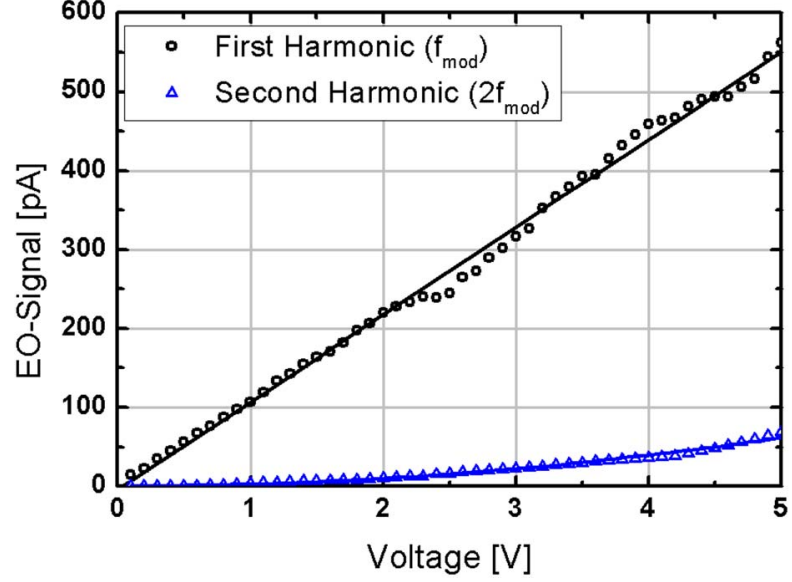

FIG. 4. (Color online) Modulation amplitude as a function of modulation voltage detected at the first (black circles) and second harmonic (blue triangles) of the modulation frequency. The lines represent linear (black) and square (blue) fits to the data points, indicating Pockels and electrical Kerr effect, respectively.

firms the observed modulation effects are indeed due to the NLO-effects of the infiltrated and poled polymer.

Using the modulation depth at the extremum points and the general properties of a Lorentzian function we calculate the index change in the polymer in the slot to be approximately $\Delta n=1.3 \times 10^{-4}$ at $1 \mathrm{~V}$ applied modulation voltage. ${ }^{18}$ The index variation due to the EO-effect is given by $\Delta n$ $=0.5 r_{33} n_{\text {poly }}^{3} E$, thus the in-device Pockels coefficient of the NLO-polymer in this sample is $9 \mathrm{pm} / \mathrm{V}$. This value is about 15 times lower than the value reported in thin films of the same material. ${ }^{15}$ We attribute this to an incomplete poling induced polar orientation of the guest chromophores in the $150 \mathrm{~nm}$ wide slot. ${ }^{7}$ This important topic is currently under investigation as this deviation indicates that the performance of the device can be significantly improved by increasing the poling efficiency in the slot. These further studies are directed toward understanding the physics of high field poling of macromolecular NLO materials in submicron silicon cavities and will be necessary to obtain an optimized poling process. Such an optimized procedure would give one order of magnitude improvement in the induced refractive index change and consequently spectral shift at a given modulation voltage.

In conclusion we fabricated slotted $\mathrm{PhC}$ waveguides to form a heterostructure cavity in SOI covered and infiltrated by NLO-polymer, which enables EO-modulation on an extremely small geometrical footprint at potentially very high modulation speeds in the gigahertz range. The devices achieved $Q$-factors of up to 2600 and we showed the EO- modulation of the devices resonance position. Our current work is directed to achieving the same large $r_{33}$ values found in bulk NLO-polymers also in a slotted $\mathrm{PhC}$ waveguide and to develop appropriate electrodes and microwave feeding schemes in order to realize modulation at gigahertz frequencies. The presented results show that slotted $\mathrm{PhC}$ waveguides in hybrid SOI and polymer materials are a possible platform for achieving ultracompact and ultrafast EO-modulators and tunable filters.

This research was supported by the German Research Foundation DFG (Grant No. EI 391/12-1). The authors acknowledge the support from CST, Darmstadt, Germany with their Microwave Studio software and Ralf Steingrüber from the Heinrich-Hertz-Institute Berlin for providing the electron beam lithography.

${ }^{1}$ L. Liao, A. Liu, J. Basak, H. Nguyen, M. Paniccia, D. Rubin, Y. Chetrit, R. Cohen, and N. Izhaky, Electron. Lett. 43, 1196 (2007).

${ }^{2}$ Q. Xu, S. Manipatruni, B. Schmidt, J. Shakya, and M. Lipson, Opt. Express 15, 430 (2007).

${ }^{3}$ X. Chen, Y.-S. Chen, Y. Zhao, W. Jiang, and R. T. Chen, Opt. Lett. 34, 602 (2009).

${ }^{4}$ M. Lee, H. E. Katz, C. Erben, D. M. Gill, P. Gopalan, J. D. Heber, and D. J. McGee, Science 298, 1401 (2002).

${ }^{5}$ T.-D. Kim, J. Luo, Y.-J. Cheng, Z. Shi, S. Hau, S.-H. Jang, X.-H. Zhou, Y. Tian, B. Polishak, S. Huang, H. Ma, L. R. Dalton, and A. K.-Y. Jen, J. Phys. Chem. C 112, 8091 (2008).

${ }^{6}$ C. Koos, P. Vorreau, T. Vallaitis, P. Dumon, W. Bogaerts, R. Baets, B. Esembeson, T. Michinobu, F. Diederich, W. Freude, and J. Leuthold, Nat. Photonics 3, 216 (2009).

${ }^{7}$ T. Baehr-Jones, B. Penkov, J. Huang, P. Sullivan, J. Davies, J. Takayesu, J. Luo, T.-D. Kim, L. R. Dalton, A. K.-Y. Jen, M. Hochberg, and A. Scherer, Appl. Phys. Lett. 92, 163303 (2008).

${ }^{8}$ T. Baehr-Jones, M. Hochberg, G. X. Wang, R. Lawson, Y. Liao, P. A. Sullivan, L. R. Dalton, A. K.-Y. Jen, and A. Scherer, Opt. Express 13, 5216 (2005)

${ }^{9}$ J. M. Brosi, C. Koos, L. C. Andreani, M. Waldow, J. Leuthold, and W. Freude, Opt. Express 16, 4177 (2008).

${ }^{10}$ A. Di Falco, L. O'Faolain, and T. F. Krauss, Appl. Phys. Lett. 92, 083501 (2008)

${ }^{11}$ J. H. Wülbern, A. Petrov, and M. Eich, Opt. Express 17, 304 (2009).

${ }^{12}$ A. Di Falco, L. O'Faolain, and T. F. Krauss, Appl. Phys. Lett. 94, 063503 (2009).

${ }^{13}$ Y. Akahane, T. Asano, B. S. Song, and S. Noda, Nature (London) 425 , 944 (2003).

${ }^{14}$ Z. Wang, N. Zhu, Y. Tang, L. Wosinski, D. Dai, and S. He, Opt. Lett. 34, 1498 (2009).

${ }^{15}$ H. Chen, B. Chen, D. Huang, D. Jin, J. D. Luo, A. K.-Y. Jen, and R. Dinu, Appl. Phys. Lett. 93, 043507 (2008).

${ }^{16}$ Available at www.cst.com.

${ }^{17} \mathrm{~J}$. Li, T. P. White, L. O'Faolain, A. Gomez-Iglesias, and T. F. Krauss, Opt. Express 16, 6227 (2008).

${ }^{18}$ M. Schmidt, M. Eich, U. Huebner, and R. Boucher, Appl. Phys. Lett. 87, 121110 (2005) 